\title{
Humidity and Temperature Monitoring System using IoT
}

\author{
Ms.Sujeetha. R/AP, K Reddy Deeraj, B Bhaskar Yeseswi, Lenin Sade
}

\begin{abstract}
Indian enterprises significantly incorporate biomedical, horticultural and pharmaceutical which are the mainstays of nation economy. The checking of temperature and humidity are significant regions for every one of these enterprises. Any sort of unbalancing in the ecological conditions or disconnected parameters can make budgetary misfortune in the profitability of pharmaceutical and horticulture enterprises. Checking of temperature and moistness are likewise required for biomedical industry for medications and cell culture strategies. In medicinal services segments, conditioncontrolled, conditions are additionally required for patients undermining. In this paper we are going to gauge temperature and humidity by utilizing Node MCU apparatus and DHT11, which will be useful for adjusting the earth to build the productivity in this in agriculture sector today's weather forecasting systems accessible based on satellite and RADAR communication. These frameworks are substantial, hard to deal with and exorbitant. They are detecting scarcely specific region and its incomplete range. In any case, in horticulture field universally not indistinguishable ecological conditions it is important to observing every single yield existing natural situation. An agriculture field premises has dissimilar humidity, temperature, moisture, light intensity because corner of plot trees and water leakage, so that kind of changes across all parameters of field are essential, and such parameters of yield continue the quality. In present paper proposed framework, enhancement of moistness and temperature. There are numerous frameworks are accessible in the market dependent on Wireless sensor organize (WSN) yet this framework is more vitality effective, little size, convenient. Sensor is coordinated bundle contains stickiness and temperature estimation ability in single bundle.
\end{abstract}

Keyword: MCU apparatus and DHT11, RADAR These frameworks are substantial, hard to deal with and exorbitant.

\section{INTRODUCTION}

There are such a large number of inserted gadgets to associate with condition by interfacing web. The addition of these sorts of articles is accomplishing the advancement of microcontroller-based frameworks which are supplanting old convoluted electronic circuits. By utilizing IoT, we can control any electronic hardware in homes and businesses. Besides, we can peruse an information from any sensor and examine it graphically from anyplace on the planet. Arduino is a microcontroller board which fills in as a little PC.

Revised Manuscript Received on December 30, 2019.

* Correspondence Author

Ms.Sujeetha. R/AP, Department of Computer science Engineering, SRM Institute of science and Technology

K Reddy Deeraj, Department of Computer science Engineering, SRM Institute of science and Technology

B Bhaskar Yeseswi, Department of Computer science Engineering, SRM Institute of science and Technology

Lenin Sade, Department of Computer science Engineering, SRM Institute of science and Technology

(c) The Authors. Published by Blue Eyes Intelligence Engineering and Sciences Publication (BEIESP). This is an open access article under the CC BY-NC-ND license (http://creativecommons.org/licenses/by-nc-nd/4.0/)
Node MCU is a stage to build up a cooperation with required programming. Arduino UNO is miniaturized scale controller unit to get an information of moistness and temperature from DHT 11 sensor and procedure it and offer it to an ESP8266 module (wi-fi module). In this paper we have various segments to follow the temperature and dampness. Segment I characterizes the moistness and temperature by utilizing stickiness and temperature sensor DHT11, area II peruses the DHT sensor module's yield and concentrates temperature and dampness esteems into an appropriate number in rate and Celsius scale, segment III framework shows mugginess and temperature on LCD, Section IV characterizes investigating and planning the framework engineering, segment $\mathrm{V}$ demonstrates the outcome and future degree

\section{EXISTING SYSTEM}

In the existing system there are many types of Humidity and Temperature monitoring system some of them are using IOT sensors, by using cloud services, by using Digital technology and Computer technology. In this paper, we are going to implement Humidity and Temperature monitoring system by using IOT. All the current working models are good by many of laboratories are losing their projects. By this project we can able to get exact value of measures present in our atmosphere More over this model in previous literature studies have been trained with other low longitude areas Temperature values and got deployed and makes it inaccurate for the recognition of Temperature and Humidity values By this project we can able to get accurate values of weather at every time

\section{PROPOSED SYSTEM}

In this Arduino Project we will figure out how to utilize the DHT11 or the DHT22 sensor for estimating temperature and moistness with the IOT hub MCU. By this project we are going to get the values to our mobiles and computers. So, we can able to know the exact values of temperature in the lab. It is easy to operate and able to understand easily. Live temperature or moistness worth is sent to a scrounge recipient through remote signal. The DHT11 sensor detects mugginess and temperature, and sends the data to computerized stick 5 of Arduino MCU, From Arduino MCU, stickiness and temperature esteems are transferred to the Cloud at regular intervals of time through ESP8266 WIFI module. From the Cloud, humidity and temperature values can be seen graphically on Firebase console platform from anywhere in the world. With the help of WIFI module we can able to access the data 


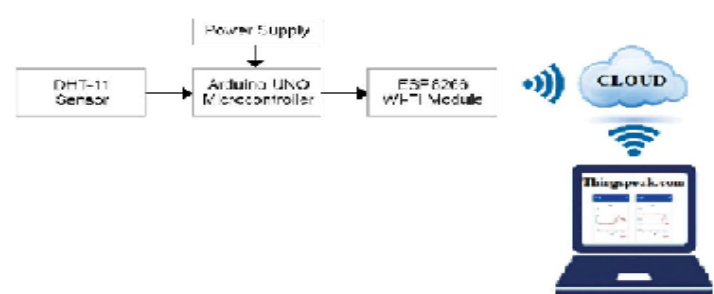

Fig (1) Block Diagram of Monitoring system

\section{RELATED WORK}

\section{ARDUINO MCU BOARD}

Arduino is another open source equipment and programming system. It needs to take consideration of an enormous innovation plan and network at moderate cost, which expands its utilization with cutting edge innovation. Arduino equipment is a motherboard for making cooperation among articles and reasonable PC programming IDE (Integrated Development Environment)

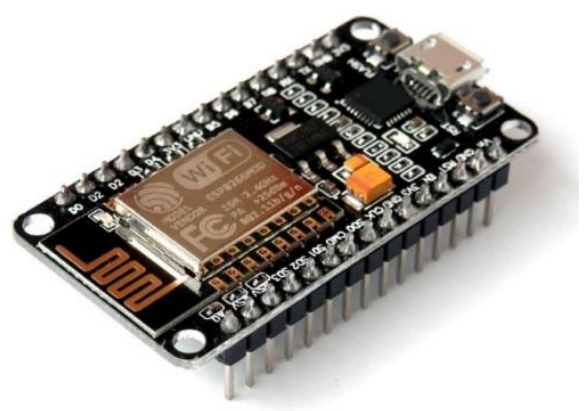

Fig (2) MCU board

\section{DHT11 Sensor}

This module includes a stickiness and temperature complex with an aligned computerized sign yield implies DHT11 sensor module is a joined module for detecting mugginess and temperature which gives an adjusted advanced yield signal. DHT11 gives us exact estimation of moistness and temperature and guarantees high unwavering quality and longhaul soundness. This sensor has aresistive sort mugginess estimation segment and NTC type temperature estimation segment with an 8-piece microcontroller inbuilt which has a quick reaction and practical and accessible in 4-stick single line package. DHT11 module deals with sequential correspondence for example single wire correspondence. This module sends information in type of heartbeat train of explicit timespan. Before sending information to Arduino it needs some introduce order with a period delay. Furthermore, the entire procedure time is about $4 \mathrm{~ms}$. The single-wire sequential interface makes framework joining speedy and simple. Its little size, low power usage and up-to-20-meter signal transmission choosing it the best choice for various applications, including those most mentioning ones. The segment is 4-stick single line stick bundle. It is advantageous to interface and uncommon bundles can be given by clients' solicitation.

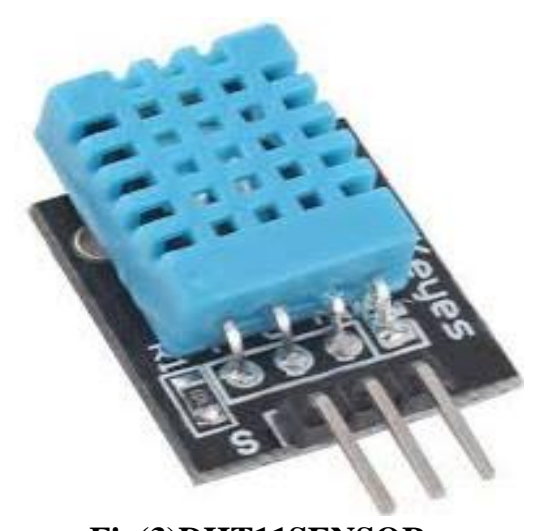

\section{WORKING}

Fig(3)DHT11SENSOR

The Temperature and Humidity Sensor Project will be controlled using an application named FIRE BASE CONTROL (Available for Android and windows) using Arduino, an Ethernet Shield and its libraries. User can securely login over Fire base control to control and monitor the room temperature and humidity. The code includes: The Arduino sketch. The Arduino sketch handles the interchanges by setting up the system. The sketch runs the program and conveys each line in turn over the server. Clients can login remotely on this web server. Utilization of DHT22 Sensor: DHT22 capacitive moistness detecting computerized temperature and dampness module is one that contains the intensify that has been adjusted carefully to flag yield of the temperature and stickiness sensors. The sensor incorporates a capacitive sensor, wet parts and a high precision temperature, estimation gadgets, and associated with a superior 8-piece microcontroller. The item has great quality, quick reaction, solid enemy of sticking capacity, and significant expense. It is certainly dependable and has incredible perseverance. Firebase console is an open source stage to store and recover an information for Internet of Things application. To utilize this, you have to enlist in Firebase cloud and afterward login to your record. After make another direct with temperature in one field and stickiness in another field as appeared. When you made another channel, it will create a two API keys, they are READ API keys and WRITE API keys. In the first place, duplicate the WRITE API keys from Firebase and glue it into the line the program. Next, supplant the Hostname and Password with your WIFI name and Wi-Fi secret key in the two lines given beneath in the program. (String Host_Name = "Pantech" and String Password = "pantech123") The Arduino program Uses DHT library, on the off chance that it isn't exhibited in your Arduino IDE, select SketchàInclude libraryàManage librariesàInstall DHT Sensor library. At that point assemble the program and transfer to an Arduino Uno through Arduino IDE. Guarantee that WiFi modem and web association in your Smartphone or PC are working appropriately. After transferred a program, the Temperature and Humidity information is transferred on Firebase Console stage. You can see it graphically in the private view window of you divert as appeared in Fig: Also, you can ready to see the transferred information

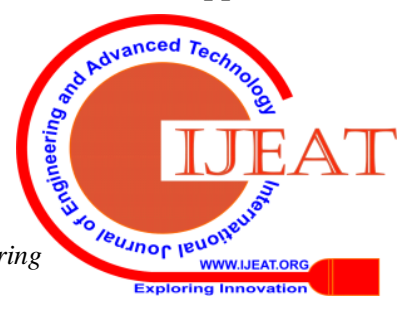


from sequential port of Arduino IDE.

Circuit outline for observing moistness and temperature is appeared in Fig. 2. It is worked around Arduino MCU, DHT11 sensor and ESP8266 Wi-Fi module. In this task, we will construct a little circuit to interface Arduino MCU with DHT11 Temperature and Humidity Sensor. One of the fundamental utilizations of interfacing DTH11 sensor with Arduino is climate observing. All the DHT11 Sensors are precisely adjusted in the research facility and the outcomes are put away in the memory. A solitary wire correspondence can be set up between any microcontroller like Arduino and the DHT11 Sensor. Also, the length of the link can be up to 20 meters. The information from the sensor comprises of fundamental and decimal parts for both Relative Humidity (RH) and temperature. The information from the DHT11 sensor comprises of 40 - bits. The DHT11 sensors have four pins, VCC, GND, information stick and a not associated stick which has no utilization. A dismantle up resistor from $5 \mathrm{~K}$ to $10 \mathrm{~K}$ Ohms is required to keep the information line high and so as to empower the correspondence between the sensor and the ArduinoMCU Board. There are a few renditions of these sensors that accompany a breakout barricades with inherent draw resistor and they have only 3 pins. A Temperature Response demonstrates a straight relationship between the real temperature (estimated by LM35) and the temperature determined from the $\mathrm{Q}$ factor of the sensor's reaction. As appeared in there is a little counterbalanced in the direct line. This is because of the distinctive temperature reaction times between the LCR sensor (the reaction time for the thermistor was $0.9 \mathrm{~s}$, however the genuine time for every estimation was $50 \mathrm{~s}$ ) and the business LM35 temperature sensor (reaction time was around $10 \mathrm{~ms}$ ), combined with the way that the estimations were recorded while the temperature was changing. Therefore, as temperature differed, the LM35, which had a marginally quicker reaction time, would have just enrolled a change while the LCR sensor was all the while recording temperature from the past minute. The LCR sensor demonstrates a direct reaction with the genuine temperature, estimated with a business temperature sensor LM35 (Texas Instruments, Dallas, TX). The goals of the deliberate temperature sensor were $0.1^{\circ} \mathrm{C}$, and the standard deviation of the estimations contrasted with the perfect line was $0.1^{\circ} \mathrm{C}$. The deliberate and real temperatures when the test chamber was over and over set at $37{ }^{\circ} \mathrm{C}$ and $40{ }^{\circ} \mathrm{C}$. These two set focuses speak to the temperature for a typical and seriously tainted human. Plots the sensor estimations at numerous cycles, demonstrating no noticeable float. Blunders in Be that as it may, we don't accept this will comprise a worry for the proposed application since disease determination doesn't require an extremely quick inspecting rate. Ordinarily, it would be sufficient to gauge the interior injury temperature for a couple of times each day to get the early beginning of contaminations. By and by, the temperature estimations can be examined at $5-10 \mathrm{~Hz}$ and after that found the middle value of to deliver a solitary information point. This will build the precision while still breaking point the estimation time to inside a couple of moments. Besides, as dictated by Romano a differential temperature estimation of $0.9{ }^{\circ} \mathrm{C}$ on the skin surfaces is touchy enough to recognize a disease at the site of a medical procedure. With averaging, the LCR sensor ought to have sufficient exactness for identifying disease. It was likewise seen that there was a little reverberation recurrence move relating to the temperature change. The temperature reliance of the reverberation recurrence was little $\left(\sim 1 \mathrm{kHz}\right.$ out of $27 \mathrm{MHz}$ for every $\left.{ }^{\circ} \mathrm{C}\right)$. This move was because of the development of the inductive windings or the center.

\section{RESULT}

The code is uploaded in the Arduino 1.8.10 software application and the Arduino MCU board were configured. In the Firebase Console the account will be created then the Data base will be created. According to the data base the Threshold values are inserted to the required temperature values and the humidity values.This make the values as a barrier when the humidity crosses its limit in a room atmosphere the notifications will be alerted and the precautions will be taken to provide the projects or plants etc.., A data base is created with the google account when we logon to the account the values are displayed .The notifications will be send to the registered mobile as the temperature and the humidity is displayed. The figures are shown below

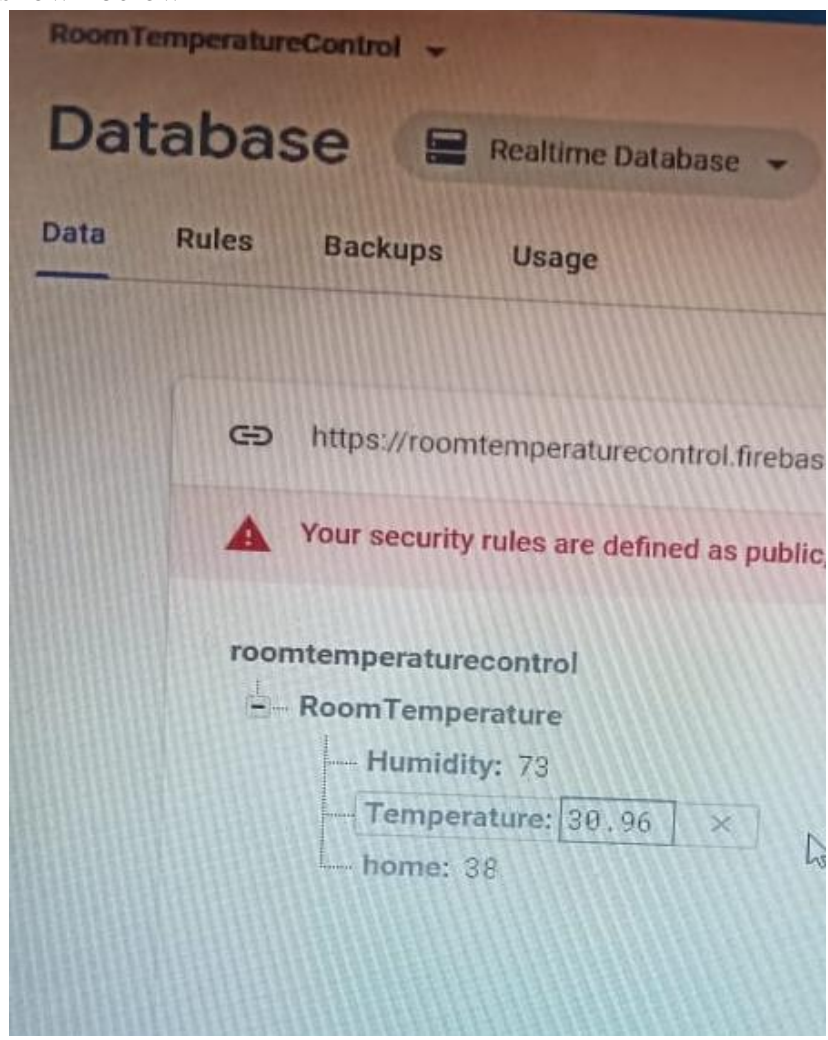

Fig (4) The values display in Database 


\section{RoomTemperatureControl}

Humidity

Humidity threshold reached!!

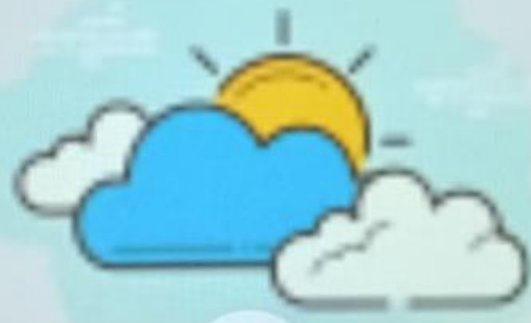

Fig (5) Notification as threshold reached in mobile

As we have discussed on the top the values of the temperature and humidity are displayed according to the process we have done. From the notification feature present in our project we can able to know when the humidity or temperature goes beyond its threshold limit.

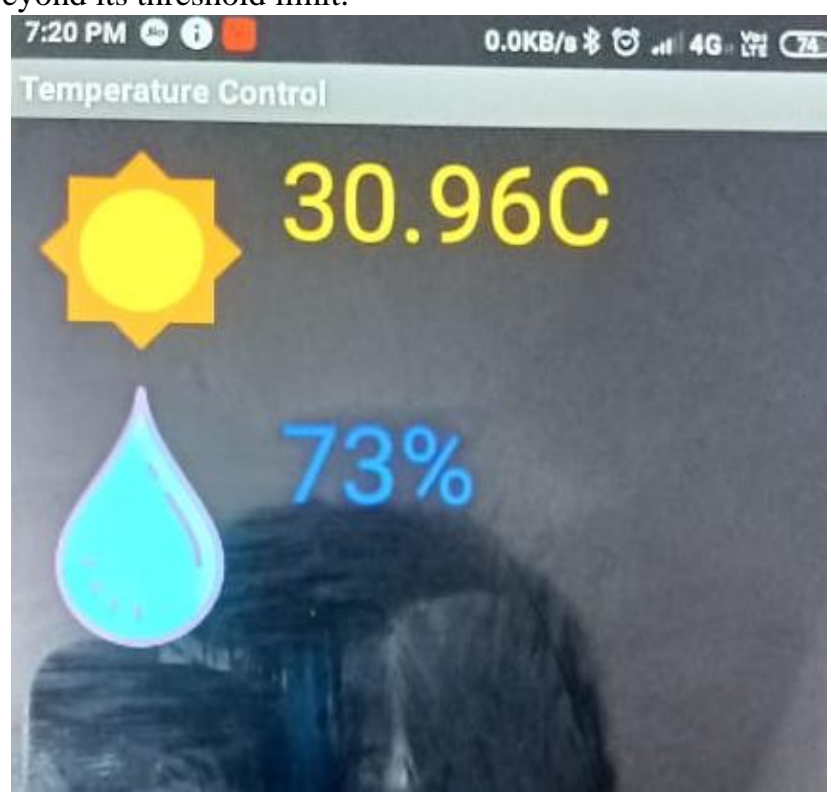

Fig (6) Display of the values in mobile

\section{LITERATURE SURVEY}

Vinayak Aappasaheb Pujari et al. have proposed the system that uses the sun-controlled power board. This system is used to screen temperature, wind speed, twist course, moistness and deluge. The distinguished data will be sent to GSM module and through section to the PC. A server is related with the database

M. Rahman Laskar et al. displayed paper on climate gauging utilizing Arduino and Cube-Sat. This proposed framework employments temperature and moistness sensor (DHT11), weight and elevation sensor (BMP180) and accelerometer (ADXL-335). The information handling unit Arduino Uno is utilized. Shape satellite is utilized to give data of climate from anyplace without utilizing organize. A gas inflatable is utilized to hold and convey the Cube satellite. This framework is easy to develop, versatile, cost productive, low power devouring and dependable. In any case, there are a few confinements, for example, gadget may not impart at long separation without amazing handset area, at higher height record of information with the assistance of gas inflatable might be an issue and parts may be harmed by downpour or long-time use Prof. C. H. Chavan et al. have proposed their framework to create remote sensor arrange for a farming domain. This framework utilizes the Wireless Sensor Networks which comprised of radio recurrence handset, sensors, microcontrollers and power sources. Equipment of this framework incorporates 8-piece AVR, ZigBee, Blue tooth module, temperature, stickiness, soil dampness sensors, LCD. This framework is solid also, effective for rural parameters observing.

\section{CONCLUSION}

IoT-Based temperature and stickiness identifying framework give an effective and complete framework for observing agrarian parameters. The remedial move can be made. IoT-Based observing of field not just enables client to lessen the human work and time, yet it likewise allows client to examine exact changes in the environment and for making conceivable move. It is less expensive in cost and expends less power. The GDP per capita in agro area can be expanded. This IoT-based framework can be reached out for controlling distinctive electronic and electrical device from remote areas and the framework can likewise reached out for soil dampness and dairy cattle checking.

\section{REFERENCES}

1. Global Journal for Innovative Research in Science and Technology (IJIRST)- Volume 1-May 2015' the Real Time Temperature Sensing utilizing Raspberry Pi

2. M. Rahaman Laskar, R. Bhattacharjee, M. Sau Giri and P. Bhattacharya, "Climate Forecasting utilizing Arduino Based Cube-Sat", Twelfth International Multiconference on Information Processing (IMCIP) - 2016

3. Vinayak Appasaheb Pujari, Mrs. M. M. Raste, Ms. A. A. Pujari, "Savvy Automatic Weather Station-a Review", International Journal of Electrical and Gadgets Engineers (IJEEE)- Vol. No. 8 Issue 01, January-June 2016

4. Prof. Satyashil Nagrale, Ms. Poonam Khetmalis, Ms. Sanika Doke, Ms. Varsha Dherange, "Constant Data Transmission for Weather Monitoring System", Global Research Journal of Engineering and Technology (IRJET)- Volume: 03 Issue: 02, Feb 2016

5. Sheik Ferdoush, Xinorong Li, "Remote Sensor Network System Design utilizing Raspberry $\mathrm{Pi}$ and Arduino for Environmental Monitoring Applications". Procedia Computer Science 34(2014) 103-110

6. Prof. C. H. Chavan, Mr. V. Karande "Wireless Monitoring of Soil Moisture, Temperature and Humidity utilizing Zigbee in Agriculture", International Journal of Building Trends and Technology (IJETT)Volume 11 Number 10 - May 2014.

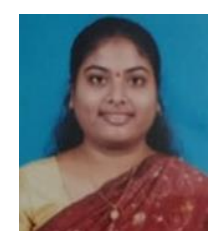

\section{AUTHORS PROFILE}

Ms. R. Sujeetha Assistant Professor, Dept of CSE, SRM Institute of Science and Technology, Chennai, India Holds a post-graduation degree M.E (CSE) from Anna University Coimbatore. Having more than three years of teaching and Industrial can be reached at Email: sujeethasrm@gmail.com

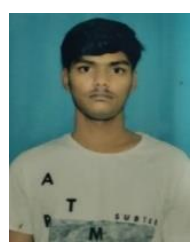

K. ReddyDeeraj, UG Scholar, B Tech, Department of Computer Science and Engineering, SRM Institute of Science and Technology, Chennai, India. He can be reached at Email: reddydeeraj31@gmail.com

Published By:

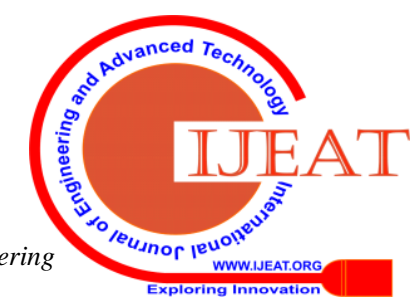




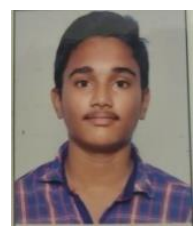

S. Lenin, UG Scholar, B Tech, Department of Computer Science and Engineering, SRM Institute of Science and Technology, Chennai, India. He can be reached at Email: Leninsade148@gmail.com

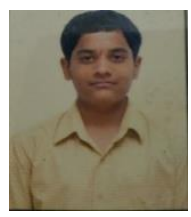

B. Bhaskar Yeseswi, UG Scholar, B Tech, Department of Computer Science and Engineering, SRM Institute of Science and Technology, Chennai, India. He can be reachedat Email:bhaskarpandu1934@gmail.com

Published By: Blue Eyes Intelligence Engineering \& Sciences Publication 